\title{
Key considerations that can make or break a closure-focused groundwater-monitoring program
}

\author{
WJ Gemson Environmental Resources Management Australia \\ TR Weaver Environmental Resources Management Australia \\ BL Heemink Environmental Resources Management Australia
}

\begin{abstract}
Potential long-term water quality impacts present one of the main challenges for effective closure of mine sites. As such, groundwater-monitoring programs form an inherent part of closure. At many mine sites, groundwater-monitoring programs develop organically over time, in response to specific issues that may arise during the life of the mine. When transitioning from operational to closure-focused groundwater monitoring the programs often undergo greater scrutiny by stakeholders. This transitioning also offers the opportunity to re-evaluate the fundamentals underpinning the program, allowing for the alignment of monitoring efforts with the site and scenario-specific monitoring and closure objectives. If gotten right significant efficiencies can be gained in achieving outcomes that support closure, but if not, quite the opposite.

This paper presents key considerations for a closure-focused groundwater-monitoring program. These include: 1) thorough assessment and confirmation of the constituents of potential concern (COPCs); 2) background concentration thresholds for COPCs that occur naturally (especially important in mineralised areas); 3) the setting of appropriate assessment criteria for water quality monitoring taking into account background concentrations and water uses; 4) developing the program within a source-pathway-receptor framework that clearly considers the environmental setting and potential changes in hydrodynamics following closure; 5) regulatory requirements; and 6) consideration of sampling frequency and temporal trends to develop trigger levels and actions to increase or decrease monitoring effort, to maintain flexibility within the program and to assist with the setting of reasonable end-points.
\end{abstract}

Keywords: water quality, groundwater, monitoring

\section{Introduction}

By its very nature, extraction and beneficiation of ore during mining leads to the physical alteration of rock, changes in geochemical conditions that geological material is exposed to and changes in hydrological dynamics at a mine site. Physical alteration is manifested by blasting, crushing and grinding, changes in geochemical conditions are generally manifested by exposing geological material to atmospheric conditions and changes in hydrological dynamics through dewatering, altered drainage patterns, water storages and water management including enhanced evaporation. These factors all have the potential to lead to deleterious impacts to water quality, and the majority of mining operations include groundwater monitoring for the assessment and management of potential impacts to groundwater.

Changes in geochemical conditions are of particular significance at mining operations where ore and host mineralogy result in the generation of acid and metalliferous drainage (AMD), which includes acid rock drainage, neutral mine drainage and saline drainage (Department of Industry, Tourism and Resources 2007), presenting a potential long-term environmental liability. AMD can manifest after a considerable latent period (International Network for Acid Prevention [INAP] 2014) and it is common for monitoring of potential AMD impacts to continue for a significant period (e.g. several decades) post-closure. Identification 
and management of potential long-term water quality impacts therefore present one of the main challenges for effective closure of mine sites.

As articulated in the Global Acid Rock Drainage Guide developed by INAP (2014), 'Monitoring is the process of routinely, systematically, and purposefully gathering information for use in management-decisionmaking.' Groundwater monitoring seeks to assess environmental changes caused by mining activities, potential impacts to receptors and the effectiveness of mitigation measures where these are in place. It is of key importance that the monitoring takes place with a clear set of objectives that are appropriate for the stage of mine life, and that monitoring data are proactively assessed to understand groundwater dynamics and chemistry for decision-making purposes. Factors that merit specific consideration when transitioning from operational to closure-focused groundwater monitoring are outlined in this paper, along with important considerations that can help establish a suitable monitoring program. For the purposes of this paper, the term 'closure-focused monitoring' captures the closure and post-closure period.

\section{Transitioning to closure-focused groundwater monitoring}

Groundwater-monitoring activities should ideally start during exploration for the purposes of establishing a baseline in mineralised but unmined areas, with monitoring continuing through the life of the mine to postclosure. The Global Acid Rock Drainage Guide (INAP 2014) presents a framework for the development of a groundwater-monitoring program, specifying that considerations include the mine plan, geological setting, geographical location (which controls the climatic conditions), the magnitude of potential AMD generation and sensitivity of the receptors. Review of the mine plan provides information on the location and scale of surface and subsurface disturbances, water management activities including dewatering, surface water diversions, water storages and application areas, ore processing and waste management activities. The aforementioned can then be used to identify sources of groundwater impact, potential migration pathways for contaminants and receptors that may be impacted by mining-related water quality impacts. This framework can then be used to assess mining-related groundwater impacts.

Closure-focused monitoring will build on the above while making sure that the monitoring aligns with the closure objectives (e.g. site-specific, and typically measurable, statements of what closure activities aim to achieve (International Council on Mining \& Metals 2019)). Key considerations for a closure-focused groundwater-monitoring programs are discussed in Section 3 . Note that these do not include specific discussion of the inclusion of data quality objectives, quality assurance / quality control (QA/QC) procedures and sound data management practices, which should form part of all groundwater-monitoring programs. In Australia, these are included and described in the National Environment Protection (Assessment of Site Contamination) Measure 1999 (the ASC NEPM) (National Environmental Protection Council [NEPC] 2013).

\section{$3 \quad$ Key considerations}

\subsection{Assessment and confirmation of constituents of potential concern}

For AMD monitoring programs the parameters that are typically of most relevance include $\mathrm{pH}$, electrical conductivity $(E C)$, oxidation-reduction potential (ORP), alkalinity, acidity, sulphate and metals and metalloids of interest. The metals and metalloids of interest are generally identified through site-specific studies, identifying those elements that may become enriched above background concentrations and potentially present a risk to receptors (INAP 2014).

Following the initial identification of the constituents of potential concern (COPCs), these could (and often do) become entrenched over time in a monitoring program. However, when transitioning from operational to closure monitoring, a groundwater-monitoring program typically undergoes greater scrutiny by stakeholders, which can lead to the long-standing consideration of COPCs being challenged. Additionally, with a long-term dataset, there may be the opportunity to reduce the list of COPCs, or the frequency of monitoring. With the rationale for the inclusion of physico-chemical parameters (such as the master 
variables on metal solubility $\mathrm{pH}$ and $\mathrm{ORP}$ ) and bulk chemistry parameters (e.g. alkalinity) generally being clear, we have seen that the onus typically falls on the set of metals/metalloids being monitored as well as organic contaminants that may be associated with activities that are ancillary to mining.

Selenium is an example of an inorganic COPC that has recently attracted significant attention by environmental authorities in order to monitor and manage its release from anthropogenic activities, with mining seen as a potential significant contributor to the release of selenium to aquatic ecosystems (Khamkhash et al. 2017). The element has the potential to bioaccumulate in aquatic ecosystems, and the removal of selenium from water is complex and often expensive, as governed by its speciation and stringent discharge limits (Stefaniak et al. 2018). Identification of whether selenium is a COPC at a specific mine site, and if it is inclusion of selenium in the groundwater-quality monitoring program, is considered prudent given the aforementioned factors.

When considering organic contaminants, perfluorooctanoic acid (PFOA) and perfluorooctanesulphonate (PFOS) have been identified as contaminants of emerging concern (CRC CARE 2014a; 2014b). PFOA and PFOS form part of a group of compounds called per- and poly-fluoroalkyl substances (PFAS), and these compounds have recently received significant attention by industry, regulators and the public. PFAS, which like selenium have the ability to bioaccumulate, have been identified in groundwater at concentrations of potential concern at numerous sites - particularly at sites where firefighting foams have been used (CRC Care 2018). Potential sources of PFAS at mine sites include firefighting training areas, workshops (with spent firefighting foams at times discharged in close proximity to workshops) and landfill sites. Other organic contaminants that may be associated with ancillary services at mines sites include petroleum hydrocarbons (typically associated with fuel spills) and chlorinated solvents (such as TCE and 1,1,1-TCA) used for metal cleaning in workshops. For sites where 1,1,1-TCA was used, the solvent stabiliser 1,4dioxane further presents an emerging contaminant of concern (Adamson et al. 2014) that may require additional assessment.

When assessing and confirming the list of COPCs, it is important to consider the nature and properties of the contaminant sources, coupled with the latest understanding of toxicological considerations and potential risk posed by COPCs to receptors. We recommend that source characterisation studies be reviewed, and that information on emerging contaminants (such as PFAS) or contaminants where the understanding of toxicology has changed (e.g. arsenic) be considered. While a monitoring program typically focused on COPCs, it is further important to include a range of analytes that assist in understanding the geochemistry at the site. These would typically include the field parameters $\mathrm{pH}, \mathrm{EC}, \mathrm{ORP}$ and temperature as well as major and minor ions.

\subsection{Evaluation of background concentration thresholds of constituents of potential concern}

In naturally mineralised regions, an understanding of background concentrations of inorganic constituents (that may ultimately become COPCs as a result of mining activities) is of key importance for the identification of actual mine impact and the establishment of appropriate assessment criteria for water quality monitoring.

Often the evaluation of background COPC concentrations is based primarily on data from monitoring locations that are clearly up-hydraulic gradient of mine features with the potential to impact on water quality, and/or on pre-mining baseline data. Background data available from these sources may, however, be lacking, especially at mine sites that have been operating for decades (with operations starting prior to a requirement or expectation that baseline conditions needed to be established) and where the landscape setting of the mine site (coupled with restrictions on off-tenement drilling access) have limited the availability of up-hydraulic gradient bores.

Where background data from these sources are lacking, one can extract a background dataset that presents background concentrations of COPCs from a site-wide groundwater-monitoring dataset. This effectively entails identifying groundwater sampled from bores that are free of mine influence. There are 
multiple advantages of extracting a site-wide background dataset from a pooled groundwater dataset including; 1) this dataset being more representative of site-wide background geochemical and hydrogeological conditions (and variability in those conditions) than a limited background dataset of offsite locations, and 2) the extracted background dataset typically being much larger in size than traditional background datasets collected from offsite locations and the larger background dataset therefore better addressing variability in background concentrations (U.S. Environmental Protection Agency [USEPA] 2014).

Such an assessment should include multiple lines of evidence to identify groundwater data representative of background conditions through the combined use of graphical and statistical tools. The first step would typically include exploratory data analysis, helping to identify important data characteristics and patterns that need to be considered in the site-specific background data evaluation. The exploratory analysis should include data quality screening (including metadata such as whether groundwater samples for metal analyses were field filtered to ensure appropriate pooling and/or conditional use of historical data), data visualisations and descriptive statistics. Examples of data visualisation tools that can form part of exploratory data analysis are shown in Figure 1.

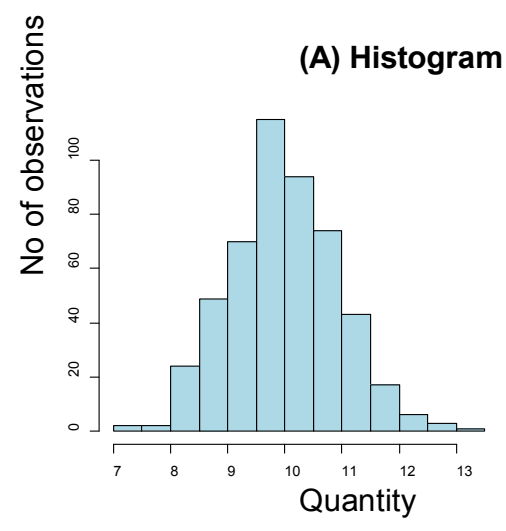

(B) Boxplots

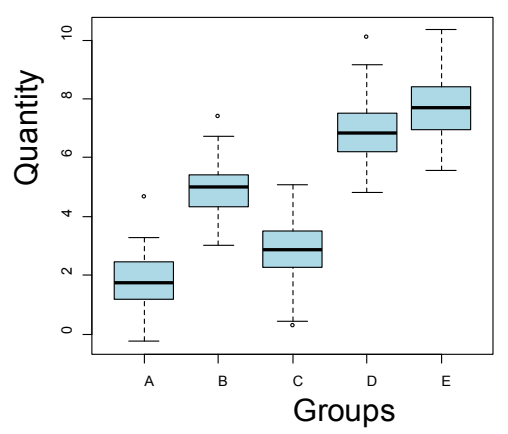

(C) Scatterplot

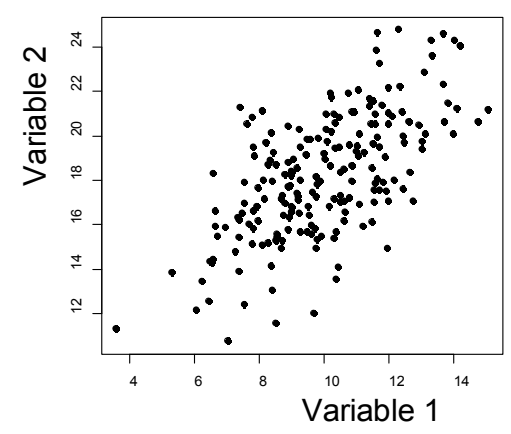

Figure 1 Data visualisation tools

Following the exploratory data analysis, the extraction of the background dataset should include temporal analysis, geochemical ratio analysis and population portioning. Temporal analyses is undertaken to evaluate whether increasing trends in COPCs are present, using both statistical methods (such as the MannKendall or Mann-Whitney test) and temporal concentration and water level plots. Increasing trends (and specifically concentration breakthrough curves) can be used to identify monitoring periods free of mine influence that are reflective of background concentrations at specific monitoring locations, and the period following the manifestation of mine impact (with the latter portion of the dataset representing samples with clear impact excluded from the background dataset).

Geochemical ratio analysis is frequently used to assess if metal concentrations in groundwater are naturally occurring (Thorbjornsen \& Myers 2007, 2008; U.S. Navy [USN] 2004). Typically this entails assessing the correlation between two elements, a trace element of interest (typically the COPC) against a major element such as aluminium or iron. With consideration of $\mathrm{pH}$, ORP and turbidity, samples that diverge from expected ratios may be identified as having been affected by mine-related impacts and can be considered for removal from the site-specific background data (with support of other lines of evidence).

Population partitioning entail the use of quantile-quantile $(Q-Q)$ plots to identify different populations in a dataset, with the presence of discontinuities (or breaks) on a normal Q-Q plot suggesting the presence of multiple populations (USEPA 2014). The iterative use of the Q-Q plots can be used to identify subpopulations, potentially distinguishing between background data and impacted locations as well as different populations within the background dataset (say for different hydrolithic units and aquifers). An example $Q-Q$ plot is presented in Figure 2, with the presence of discontinuities in a normal $Q-Q$ plot suggesting that the dataset may represent multiple populations. 


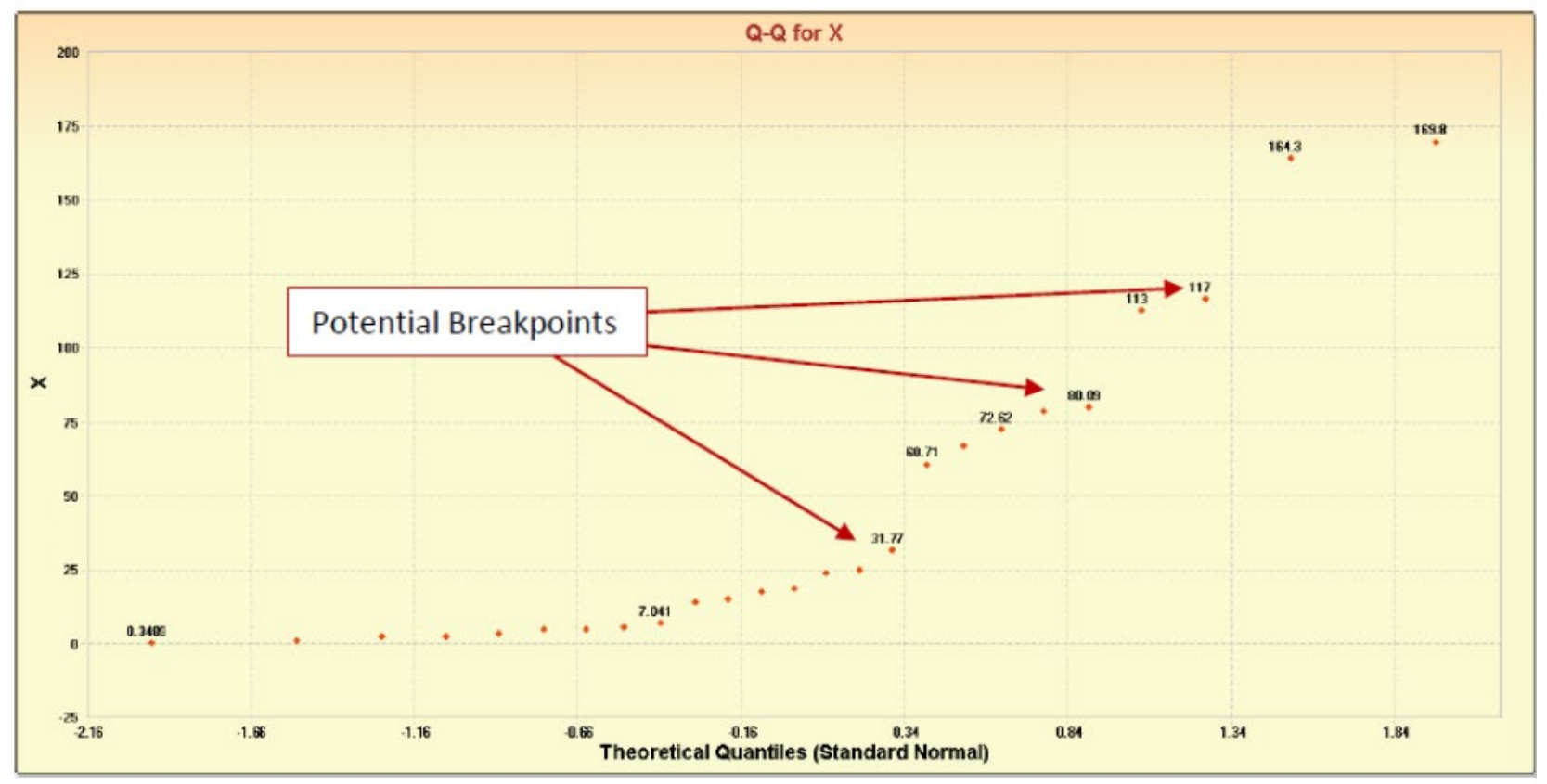

Figure 2 Example Q-Q plot showing potential breakpoints (from USEPA 2014)

While the temporal analyses, geochemical ratio analysis and population partitioning described above would typically provide sufficient lines of evidence for the identification of a background dataset, there are additional statistical tests that could be utilised. This includes multivariate statistical methods that consider groups of constituent concentrations concurrently to identify unique chemical mixtures. Multivariate tools provide a snapshot of all the data, which could make it easier to detect patterns in suites of background fingerprints across the site-a data view that is hard to obtain when evaluating each analyte in isolation. Using multivariate techniques, it may be possible to associate each sample in the dataset with a specific source or as a blend of multiple sources. Samples that appear enriched in a particular element can then be mapped spatially to assess whether it should be included in the background dataset.

Importantly, the outputs of the statistical methods need to be integrated with all available lines of evidence to finalise the background dataset. This includes interpreting the statistical results in the context of the historic and current geochemical and hydrogeological conceptual model of the site and challenging the conceptual model if the results of the statistical analysis provide sufficient justification to do so.

Once the background dataset has been identified and extracted from the pooled dataset, upper limits of background concentrations can be established. Typically, this would be done through background threshold values or percentiles (e.g. 95th percentile) to characterise the upper threshold of background concentrations. A percentile based threshold approach can however lead to a high incidence of 'false positives' when assessing whether monitoring data exceed background values. For this reason alternative statistical methods are recommended when the size of the monitoring dataset being compared to background values is greater than 15 detected observations and the frequency of detects is greater than 60 percent (USEPA 2015, USN 2004). These alternative statistical methods include hypothesis testing (for example the nonparametric Wilcoxon Rank Sum test, which is a test of central tendency [medians] between two datasets, or the slippage test, which is a nonparametric test of 'upper' tails [e.g. high concentrations] between two datasets) and/or upper simultaneous limits. The selection of the most appropriate method for deriving background concentrations is recommended after the background dataset has been established to ensure that an appropriate method is utilised. 


\subsection{Contextualisation of program within source-pathway-receptor framework}

As per standard risk-based management practices, the monitoring program should be contextualised within a source-pathway-receptor (SPR) linkage framework. Examples of typical potential sources at mine sites, pathways and receptors are presented in Figure 3.

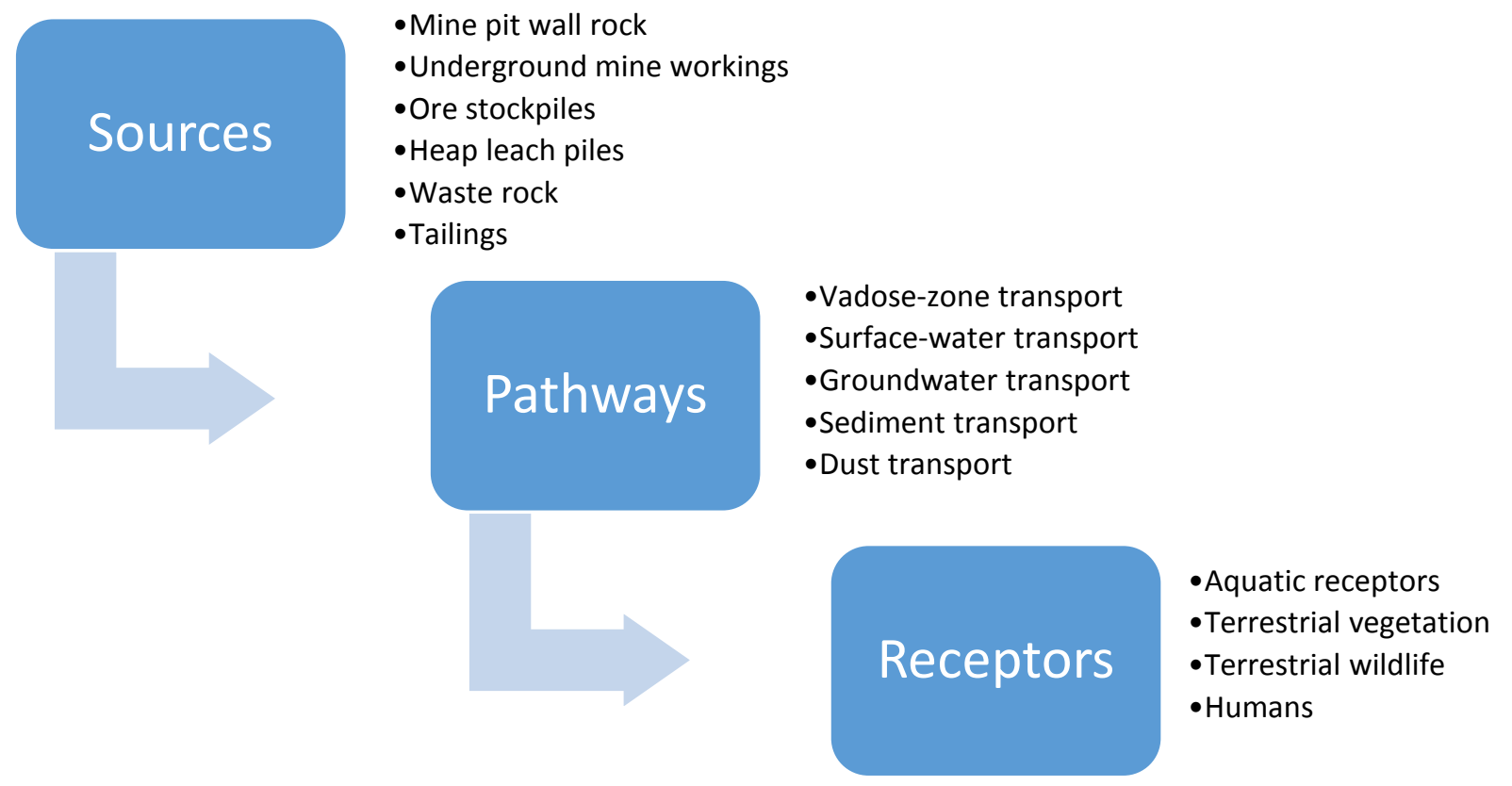

Figure 3 Typical sources, pathways and receptors for water quality impacts

At mine sites the primary sources of AMD typically include mineral waste (waste rock and tailings) and reactive wall rock within pits and underground mine workings. The primary pathway for transport of impact (acidity, elevated metals, metalloids and salinity) associated with these sources is the flow of water, with transport occurring through infiltration through the vadose zone, surface water and groundwater flows. In comparison to water based transport, sediment and air pathways are typically less significant. With water being the primary pathway, impact to aquatic ecosystems, including groundwater-dependent ecosystems and human groundwater users, typically includes the primary receptors of interest (INAP 2014).

For closure-focused monitoring, it is of key importance to consider changes in hydrodynamics associated with closure that can materially affect the SPR linkages at a mine site. These changes in hydrodynamics could include cessation of dewatering leading to the flooding of mine workings, with changes in dewatering potentially leading to reversals in hydraulic gradients and groundwater flow directions in the long-term. For example, at an underground gold mine in Western Australia that we assessed, dewatering has created a drawdown cone with a radius of influence that extends to the tailings storage facilities (TSFs) that are showing signs of significant leakage. Dewatering water is being treated, and the dewatering cone effectively captures poor quality seepage from the TSFs. Following closure and cessation of the dewatering activities, pre-mining hydraulic gradients and flow directions are expected to eventually re-establish with indications that this may lead to off-tenement migration of groundwater-quality impacts (and an SPR linkage that significantly differs from the mine operational period). Consideration of such changes in hydrodynamics following closure and the resultant implications for potential SPR linkages are extremely important for close focused groundwater-monitoring programs.

\subsection{Establishment of assessment criteria}

The establishment of assessment criteria needs to take into consideration the SPR framework for the area being monitored, the associated environmental values identified and the long-term mine closure goals. The criteria may then also need to consider background concentration thresholds for COPCs that are naturally 
occurring so that, for instance, generic aquatic ecosystem water quality criteria that have concentrations below naturally occurring COPC concentrations are not applied as assessment criteria.

The site-relevant and goal-specific criteria then essentially provide the long-term water quality goals for groundwater and receiving environments in the context of potential mine influence. These criteria can then provide the basis to assess whether monitoring results indicate that the site is on track to meet long-term closure goals, and to inform when ongoing monitoring can be reduced or ceased.

\subsection{Compliance with regulatory requirements}

All groundwater-monitoring programs, including post-closure-focused monitoring programs, need to comply with local jurisdictional regulatory requirements that are in place. This may include specific requirements for sampling methods, sampling frequency, quality assurance and quality control programs, laboratory analysis standards and accreditations as well as the COPCs included in monitoring.

In our experience, regulatory requirements are seldom overly prescriptive when looking at sampling frequency and the COPCs included in monitoring, rather allowing for site-specific decision-making on these aspects of a monitoring program. That said, regulatory requirements for the assessment of contaminated land and risk assessments will likely underpin the assessment methodology that leads to the establishment of the COPCs monitored as well as sampling frequency. In Australia, this is represented by the ASC NEPM (NEPC 2013) and is underpinned by the SPR framework established for the site.

In countries where no specific regulatory requirements are in place, following the general direction provided in guidance documents such as the Global Acid Rock Drainage Guide (INAP 2014) and MEND guidance (e.g. Robertson et al. 1990) would demonstrate alignment with industry practice.

\subsection{Consideration of sampling frequency and temporal trends to develop trigger levels and actions}

Sampling frequency is an important consideration for the evaluation of temporal trends. As described by Robertson et al. (1990), efficient monitoring programs should be stratified according to the dominant pattern of variation in the sampling data. This requires an understanding of climatic conditions at the mine site. For example, in environments with strong seasonal rainfall patterns, seepage from WRDs or ore stockpiles may be intermittent and the preferred timing of water quality monitoring may be dependent on rainfall events (INAP 2014). Mine sites in the Pilbara in Western Australia and mines located in areas of the Northern Territory in Australia are cases in point, where the majority of rainfall is associated with localised thunderstorms and cyclonic events occurring in summer and early autumn. Accumulated sulphide oxidation products would typically be flushed with the first major rainfall event, and to capture the first flush event it is helpful for the associated sampling event to be flexible in timing. Near continuous monitoring of water quality parameters such as EC as well as groundwater levels through the use of loggers in water sampling locations can also be of significant value when monitoring flushing and dilution associated with large rainfall events.

As articulated by Weaver et al. (2013) monitoring programs further need to consider climate variability, particularly over the closure period. Extended drought, major floods, and changing weather patterns can alter recharge and discharge areas, oxidation profiles, and ultimately, contaminant flux from mine sites. Unless these effects are considered in the spatial and temporal design of the monitoring program, the resultant program may fail to capture data that are critical to evaluating environmental impacts from mine sites. It is therefore recommended that the development of long-term monitoring programs include an assessment of potential climate variability impacts on groundwater dynamics and that the program includes adaptive elements so that targeted or revised monitoring can be implemented.

Once a closure-focused monitoring program is in place, temporal trends in solute concentrations need to be evaluated at regular intervals (potentially annually or at critical climatic periods in the early stages of post-closure monitoring). These trends can be assessed using a combinations of graphical and statistical 
trend assessment tools, with common nonparametric tools including the Mann-Kendall and the MannWhitney test (with the Mann-Whitney test typically being used where seasonal trends in solute concentrations are evident). The temporal trend assessment results can then be used to evaluate predicted modelling simulations and the effectiveness of mitigation measures.

Trigger values associated with long-term monitoring are recommended to have both 'alert' and 'response' actions. Concentrations that exceed an 'alert' value would likely trigger increased monitoring and assessment (e.g. frequency, location, analytes), while concentrations exceeding a 'response' value would trigger contingency measures to be initiated (INAP 2014). In addition to concentration values, it is also valuable to tie the so called 'alert' triggers to identified increases in concentrations of COPCs (with the increases ideally tied to statistical significant increases, set at a level of statistical significance [e.g. 90\%] that is considered appropriate by stakeholders). In addition to triggers for increased monitoring and assessment or the implementation of mitigation measures, the closure-focused monitoring program should have triggers for declining, and ultimately cessation of, monitoring as supported by trend assessment outcomes and monitoring compared to closure goals.

\section{Conclusion}

Transitioning from operational to closure-focused groundwater monitoring presents an opportunity to reevaluate the fundamentals underpinning the monitoring program and thus allow for the alignment of monitoring efforts with the site- and scenario-specific monitoring and closure objectives. Key considerations for closure-focused monitoring are outlined in this paper and include 1) thorough assessment and confirmation of the COPCs; 2 ) background concentration thresholds for COPCs that occur naturally; 3 ) the setting of appropriate assessment criteria for water quality monitoring, taking into account background concentrations and water uses; 4) developing the program within an SPR framework that clearly considers the environmental setting and potential changes in hydrodynamics following closure; 5) regulatory requirements; and 6) consideration of sampling frequency and temporal trends to develop trigger levels and actions to increase or decrease monitoring effort. Consideration of the aforementioned can lead to the development and implementation of a fit for purpose and adaptive monitoring program that aligns with site-specific closure objectives.

\section{Acknowledgement}

We would like to acknowledge Mark Shibata and Natasha Hausmann, our United States based colleagues, for contributing to the approach outlined in the evaluation of background concentrations section through consulting work on which we have collaborated. We would further like to acknowledge the contributions of the paper reviewers, whose review comments contributed to the refinement of this conference paper.

\section{References}

Adamson, DT, Mahendra, S, Walker, KL, Rauch, SR, Sengupta, S \& Newell, CJ 2014, 'A Multisite Survey To Identify the Scale of the 1,4Dioxane Problem at Contaminated Groundwater Sites', Environmental Science \& Technology Letters, vol. 1, pp, $254-258$.

CRC CARE 2014a, Development of guidance for contaminants of emerging concern, CRC CARE Technical Report no. 32, CRC for Contamination Assessment and Remediation of the Environment, Adelaide.

CRC CARE 2014b, Environmental impact of priority contaminants: A literature review, CRC CARE Technical Report no. 29, CRC for Contamination Assessment and Remediation of the Environment, Adelaide.

CRC CARE 2018, Practitioner guide to risk-based assessment, remediation and management of PFAS site contamination, CRC CARE Technical Report no. 43, CRC for Contamination Assessment and Remediation of the Environment, Newcastle.

Department of Industry, Tourism and Resources 2007, Managing Acid and Metalliferous Drainage, Commonwealth of Australia.

International Network for Acid Prevention 2014: Global Acid Rock Drainage Guide. Version number 1.0, http://www.gardguide.com/index.php/Main_Page

International Council on Mining \& Metals 2019, Integrated Mine Closure: Good Practice Guide, 2nd edn.

Khamkhash, A, Srivastava, V, Ghosh, T, Akdogan, G, Ganguli, R \& Aggarwal, S 2017, Mining-Related Selenium Contamination in Alaska, and the State of Current Knowledge, Minerals 2017, vol. 7, issue 3, p. 46.

National Environmental Protection Council 2013, National Environmental Protection (Assessment of Site Contamination) Measure 1999, National Environment Protection Council, Canberra. 
Robertson, E, Robertson, S \& Kirsten, BC 1990, Monitoring Acid Mine Drainage, Mine Environmental Neutral Drainage Program Project 4.7.1.

Stefaniak, S, Dutta, A, Verbinnen, B, Shakya, M \& Rene, ER, 2018, 'Selenium removal from mining and process wastewater: a systematic review of available technologies', Journal of Water Supply: Research and Technology-Aqua, vol. 67, issue 8, pp. 903-918.

Thorbjornsen, K \& Myers, J 2007, 'Identifying Metals Contamination in Groundwater Using Geochemical Correlation Evaluation. Environ', Forensics, vol. 8, issue 1-2, pp. 25-35.

Thorbjornsen, K \& Myers, J 2008, 'Geochemical Evaluation of Metals in Groundwater at Long-Term Monitoring Sites and Active Remediation Sites', Remediation, vol. 18, issue 2, pp. 99-114.

U.S. Environmental Protection Agency 2014, Extracting site-specific background dataset for a constituent from a broader dataset consisting of onsite constituent concentrations \& estimating background level constituent concentrations.

U.S. Environmental Protection Agency 2015, ProUCL Version 5.1: Technical Guide, EPA/600/R-07/041, Office of Research and Development.

U.S. Navy 2004, Guidance for Environmental Background Analysis. Volume III: Groundwater, UG-2059-ENV, Naval Facilities Engineering Command, Washington.

Weaver, TR, Price, AD, Patterson, O \& Gemson, WJ 2013, 'Developing Resilient Water Monitoring Programs in Changing Climate Conditions', Proceedings of the Water in Mining 2013 Conference, Australasian Institute of Mining and Metallurgy, Carlton. 
